\title{
Reflexiones sobre la
}

infraestructura y el uso de sistemas inteligentes en la logística

Memorias del I Seminario Internacional de Logística

Giovanny Alexander Baquero Villamil Francisco Javier Caballero Otálora Javier Plutarco Castañeda Torres Andrés Ignacio Zamudio Castro

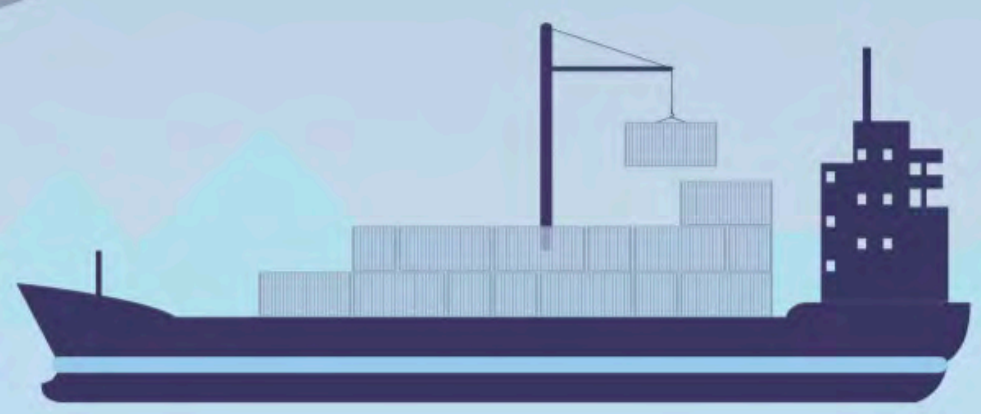




\section{REFLEXIONES SOBRE LA}

\section{INFRAESTRUCTURA Y EL USO}

\section{DE SISTEMAS INTELIGENTES \\ EN LA LOGÍSTICA}

MEMORIAS DEL I SEMINARIO INTERNACIONAL DE LOGÍSTICA

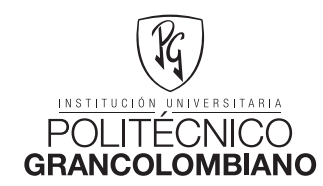

Giovanny Alexander Baquero Villamil

Francisco Javier Caballero Otálora Javier Plutarco Castañeda Torres Andrés Ignacio Zamudio Castro 
(c) Institución Universitaria Politécnico Grancolobiano

Reflexiones sobre la infraestructura y el uso de sistemas inteligentes en la logística.

Memorias del I seminario internacional de logística

ISBN: 978-958-8721-59-0

E-ISBN: 978-958-8721-46-0

Septiembre de 2017

Bogotá, Colombia

Editorial Politécnico Grancolombiano Av. Caracas No. 63-55, Piso 4

PBX: 7455555 Ext. 1171

editorial@poligran.edu.co

\section{Facultad de Ingeniería y Ciencias Básicas}

\section{Autores}

Giovanny Alexander Baquero Villamil Andrés Ignacio Zamudio Castro

Institución Universitaria Politécnico Grancolombiano

Francisco Javier Caballero Otálora

Corporación Universitaria Iberoamericana

Javier Plutarco Castañeda Torres

Fundación Universitaria del Área Andina

Lider de Publicaciones

Eduardo Norman Acevedo

Analista de Producción Editorial

Paulo Mora Noguera

Corrección de Estilo

Hernán Darío Cadena

Baquero Villamil, Giovanny Alexander
Reflexiones sobre la infraestructura y el uso de
sistemas inteligentes en la logística: memorias del I
seminario internacional de logística / Giovanny
Alexander Baquero Villamil; Francisco Javier
Caballero Otálora; Javier Plutarco Castañeda Torres
y Andrés Ignacio Zamudio Castro; lider de
publicaciones, Eduardo Norman Acevedo. - Bogotá
D.C.: Editorial Politécnico Grancolombiano, 2017.
66 p.; 14.6x 22.2 cm.
Incluye referencias bibliográficas.
ISBN: $978-958-8721-59-0$
E-ISBN: $978-958-8721-46-0$
1. Transporte -- Logística --Colombia 2.
Transporte por carretera -- Colombia 3. Río
Magdalena - Nave- gabilidad -- Colombia 4.
Transporte-- Tecnologías de la información y
comunicación. TIC 5. Transporte -- Congresos,
conferencias. I. Norman Acevedo, Eduardo. II.
Institución Universitaria Politécnico
Grancolombiano. Facultad de Ingeniería y
Ciencias Básicas. III. Tít.
SCDD 388.04 Institución Universitoria Politécnico Grancolombiano.

\section{Armada Electrónica}

Santiago Arciniegas Duarte

\section{Impresión}

Xpress Estudio Gráfico y Digital S. A.

\section{¿Cómo citar este Libro?}

Baquero Villamil, G. A., Caballero Otálora, F. J., Zamudio Castro, A. I., \& Castañeda Torres, J. P., (2017), Reflexiones sobre la infraestructura y el uso de sistemas inteligentes en la logística. Memorias del I seminario internacional de logística, Bogotá: Editorial Politécnico Grancolombiano.

La editorial Politécnico Grancolombiano pertenece a la Asociación de Editoriales Universitarias de Colombia, ASEUC.

El contenido de esta publicación se puede citar o reproducir con propósitos académicos siempre y cuando se dé cuenta de la fuente o procedencia. Las opiniones expresadas son responsabilidad exclusiva de los autores. 


\section{Tabla de contenido}

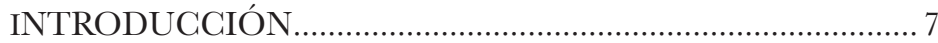

CAPÍTULO I: Navegabilidad del río Magdalena ......................... 10

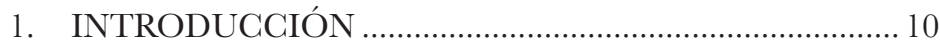

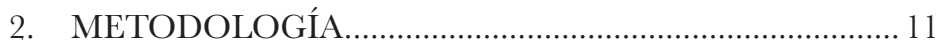

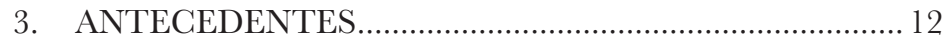

4. PROYECTOS DE DESARROLLO EN

INFRAESTRUCTURA FLUVIAL DEL RÍO

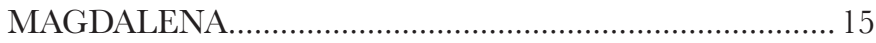

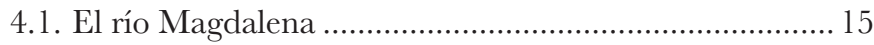

4.2. Estado actual del corredor fluvial y sus puertos............. 16

4.3. Puertos Fluviales ............................................................ 17

4.4. CONPES 3758: plan para restablecer la navegabilidad

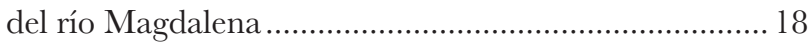

4.5. Proyectos de desarrollo en infraestructura ....................... 20

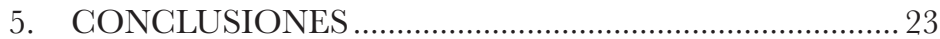

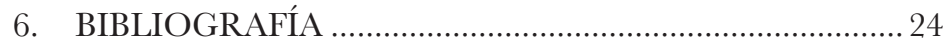

CAPÍTULO II: Aplicación de los sistemas inteligentes de información en transporte por carretera de equipo petrolero ....... 29

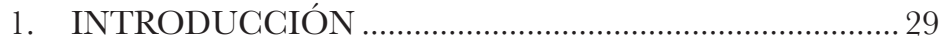

2. EL TRANSPORTE GARRETERO EN COLOMBIA ....... 30

3. SISTEMAS INTELIGENTES DE TRANSPORTE ............33

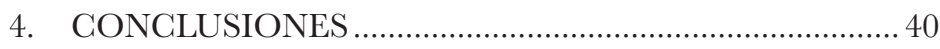

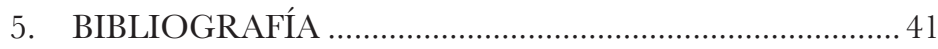


CAPÍTULO III: Tecnologías de la información y las

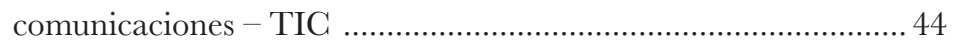

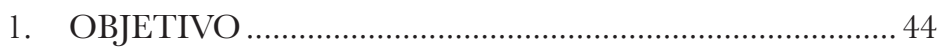

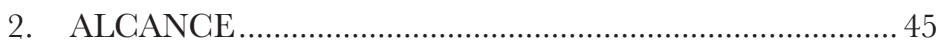

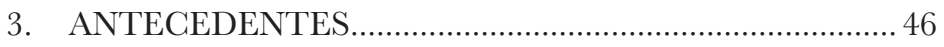

4. PLAN NAGIONAL DE TEGNOLOGÍAS DE LA INFORMACIÓN Y LAS COMUNICACIONES (PNTIC)

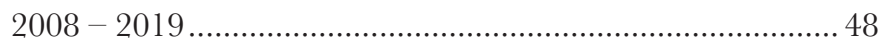

5. INDICADORES DEL SUBSISTEMA DE TEGNOLOGÍAS DE LA INFORMACIÓN Y LAS GOMUNICACIONES -

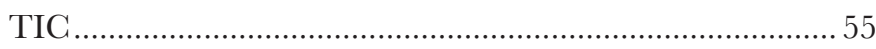




\section{INTRODUCCIÓN}

Las tecnologías de la información y las comunicaciones son cada vez más importantes y determinantes en el desarrollo económico y social de un país. Es así como por medio de diferentes estudios encabezados por la Unión Internacional de Comunicaciones se realiza la correlación entre la penetración del internet y la tasa de crecimiento del PIB (UIT, 2012). Adicionalmente son un medio para lograr el cumplimiento de los objetivos del milenio, específicamente en la erradicación de la pobreza, la universalidad de la educación, la equidad de género, la salud infantil entre otros (UIT, 2014).

Es así como las TIC son transversales en el desarrollo y se encuentran integradas directamente con la logística, ya que por medio de esta se han implementado procesos cada vez más eficientes que les permiten a las empresas ser más competitivas sin importar su tamaño. Adicionalmente, mediante su integración en la logística se ha logrado desarrollar nuevos mercados, como el e-commerce, llegando a cumplir con las expectativas de los clientes en el lugar correcto, 
al tiempo correcto, en el estado correcto, al costo correcto y con el producto correcto.

En este orden de ideas, la Corporación Universitaria Iberoamericana, la Institución Universitaria Politécnico Grancolombiano, la Fundación Tecnológica Colombo-Germana y la Fundación Universitaria del Área Andina tomaron como eje central del I Seminario Internacional de Logística "La logística como aliado estratégico del e-commerce" para analizar desde un punto de vista profesional y académico la forma en que se pueden cumplir los objetivos organizacionales por medio de la utilización de las TIC en un campo específico como es el de la logística, permitiendo a las empresas alcanzar la globalización ${ }^{1}$ de una forma integral al tener el flujo continuo en todos sus procesos, permitiendo la trazabilidad tanto de los productos como de los medios de transporte, pero para poder cumplir con los objetivos de la logística de relacionar a proveedores con los clientes con un costo logístico adecuado es necesario contar con los factores que le permiten su desarrollo, entre los que se encuentra la Infraestructura, la cual se ha determinado como una de las causas del bajo desempeño logístico, el cual ubica al país en el puesto 108 según el Foro Económico Mundial para el año 2014-2015 (WEF, 2014), ya que aún no se ha logrado desarrollar en el país el transporte multimodal, especialmente aprovechando el modo de transporte fluvial por el rio Magdalena. Adicionalmente se evidencia un deterioro en materia de calidad de la infraestructura frente a referentes internacionales. Es así como desde el Consejo Privado de Competitividad se realizan recomendaciones para desarrollar nuevos modos de transporte y establecer plataformas logísticas (Consejo Privado de Competitivi-

1 Globalización: es un término que guarda estrecha relación con la palabra integración (integración de países, regiones, mercados, economías, costumbres, etc.), y es un proceso que se ha venido dando a nivel mundial, en el cual muchos de los aspectos de la vida humana de unos lugares se han ido relacionando e interconectando con los de otros y, en general, con el mundo entero. 
dad, 2015), que le permitan al país una mayor competitividad en el campo internacional; este tema también fue analizado en el marco del I Seminario Internacional de Logística.

Bibliografía

- Cámara Colombiana de la Infraestructura. (2014). Colocación de Bonos y Financiamiento. Bogotá: Infraestructura y Desarrollo.

- Consejo Privado de Competitividad. (2015). Informe Nacional de Competitividad 2014-2015. Bogotá: Zetta Comunicaciones.

- UIT. (2012). Impact of Broadband on Economic. Geneva: Unesco.

- UIT. (2014). The state of broadband: Broadband. Geneva: Unesco.

- WEF. (2014). Global Competitiveness Report. Geneva: World Economic Forum. 